\title{
Null-Result Detection and Einstein-Podolsky-Rosen Correlations
}

\author{
Luiz Carlos Ryff \\ Instituto de Física, Universidade Federal do Rio de Janeiro, \\ Caixa Postal 68528, 21041-972 RJ, Brazil \\ E-mail: ryff@if.ufrj.br
}

August 31, 2018

\begin{abstract}
It follows from Bell's theorem and quantum mechanics that the detection of a particle of an entangled pair can (somehow) "force" the other distant particle of the pair into a well-defined state (which is equivalent to a reduction of the state vector): no property previously shared by the particles can explain the predicted quantum correlations. This result has been corroborated by experiment, although some loopholes still remain. However, it has not been experimentally proved - and it is far from obvious - that the absence of detection, as in null-result (NR) experiments could have the very same effect. In this paper a way to try to bridge this gap is suggested. As already shown for the case of Einstein-PodolskyRosen (EPR) correlations, if NR detections cannot induce a reduction of the state vector, then faster-than-light (FTL) communication becomes possible, at least in principle. But, it will be demonstrated that - as entertained by Bohm - this does not necessarily lead to a causal paradox, or to the rejection of the Lorentz transformations.
\end{abstract}

\section{Introduction}

In a null-result (NR) experiment, instead of registering the presence of a particle, a detector "registers" its absence [1]. In some situations, since there is no detector "click," it is possible to know the path that the particle has not followed and, by exclusion, we can also infer the path followed by the particle. Naturally, from the habitual standpoint there seems to be nothing mysterious in this. On the other hand, although they involve no irreversible amplification (that is usually associated with the measurement process [2]), it is accepted that NR detections have the same capability of reducing (or collapsing) the quantum state vector as ordinary $(\mathrm{O})$ detections. However, as has already been argued, the experiments so far discussed can be explained without the need to invoke any collapse [3]. It is also noteworthy that although the experimental violations of Bell's inequalities [4] corroborate the point of view according to which the detection of a particle of an entangled pair "forces" the other distant particle of the pair into a well-defined state (more specifically, no property previously shared by the particles can explain the observed quantum correlations), it has 
not been proved-and it is far from obvious-that the absence of detection, as in NR measurements, could have the very same effect. Therefore, the subject, although relatively old, is far from being settled. A way of trying to bridge this gap will be suggested here. This approach differs from previous ones $[5,6]$ in two aspects: (1) time-like events will be considered (and the importance of doing so will be stressed), and (2) coincident detections will be taken into account, which simplifies the experiment and makes the differences between the predictions more accentuated, based, on the one hand, on the assumption that NR detection induces a collapse and, on the other, on the assumption that NR detection does not induce a collapse (at least as it is usually understood). As already shown $[5,6]$, if NR detections cannot induce, via Einstein-PodolskyRosen (EPR) correlations [7], a reduction of the state vector, then, faster-thanlight (FTL) communication (an idea entertained by Bohm [8]) becomes possible, at least in principle. In this paper it will be demonstrated that, strange as it may sound, this can be consistent with Lorentz transformations, not leading to any causal paradox.

To see the importance of considering time-like events, a point that has been overlooked in previous discussions on NR detection, let us consider the following simple experiment. A single photon (from a pair generated via spontaneous parametric down-conversion (SPDC) [9], for instance), impinges on a 50:50 beam splitter. A first detector is placed near the beam splitter to register a reflected photon, and a second one is placed distant from the beam splitter to register a transmitted photon. Whenever the first detector does not click, it is possible to infer that the photon has been transmitted. From a quantum mechanical standpoint, it can be said that the absence of detection induced a reduction of the state vector, "forcing" the photon into the transmitted state. However, the lack of detection at the first detector and the detection at the second are space-like events [10]; therefore, there are an infinite number of Lorentz frames in which the second detector clicks before the first has not clicked. Hence, it can equally be said that the detection of the second photon induced the collapse of the reflected state. Consequently, this cannot be considered an indisputable NR-detection measurement.

Actually, the above example also admits a simple interpretation: in an ideal situation, if a particle has not been reflected, it has necessarily been transmitted [11]. To avoid this sort of explanation, it is important to consider an experiment that violates a Bell inequality, namely one in which an objective (as opposed to subjective) change of probability must necessarily occur [12].

As has been pointed out $[3,6]$, in the Gedanken experiments discussed by Renninger [13] and Dicke [14] the lack of detection, despite giving us information about the state of the system, does not necessarily imply that the system has been forced into this state by this very lack of detection. The same is true, for example, when we infer, from the absence of resonance fluorescence, that a quantum jump has occurred [3]. Moreover, it is always possible to describe these experiments in another Lorentz frame in which the reduction of the state vector has not been induced by the absence of detection. Therefore, it seems fair to say that no unquestionable collapse-inducing NR-detection measurement has been performed since the emergence of quantum mechanics (QM), more than eighty years ago. Naturally, NR-detection reduction of the state vector is far from being a trivial fact, and its experimental verification is extremely important for the investigation of the foundations of QM [15]. 


\section{The experiment}

Let us consider the experiment represented in Fig.1, which is a variant of an experiment performed by Aspect, Grangier, and Roger [16]. To have a clear understanding of this proposal, we will initially examine the ideal situation. A source $S$ generates pairs of photons $\left(\nu_{1}, \nu_{2}\right)$ in the state

$$
|\psi\rangle=\frac{1}{\sqrt{2}}\left(|a\rangle|a\rangle+\left|a_{\perp}\right\rangle\left|a_{\perp}\right\rangle\right)
$$

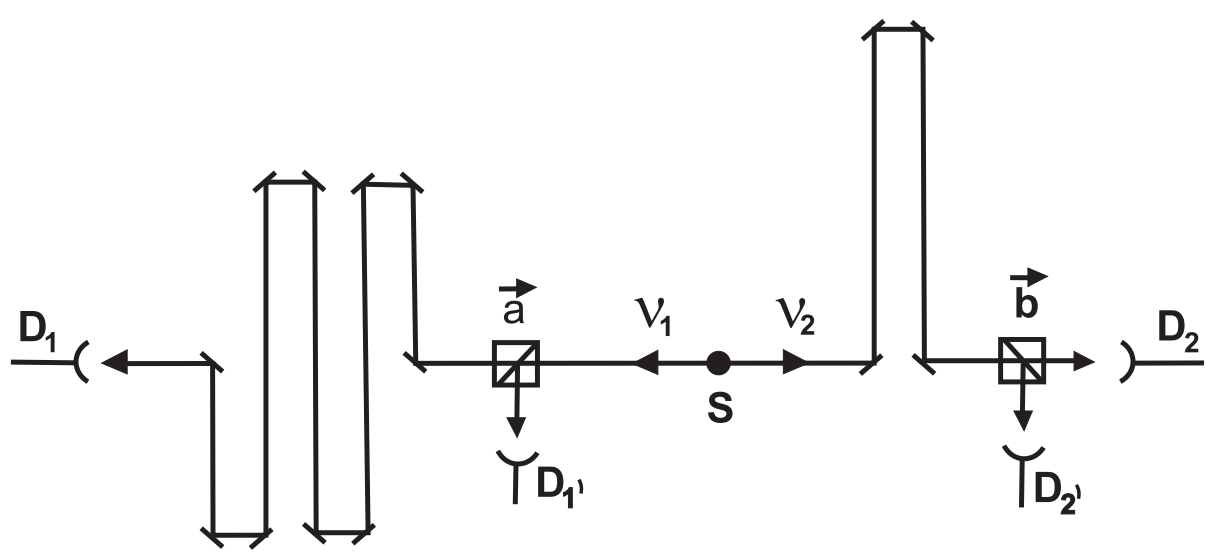

Fig.1: The proposed experiment: whenever $\nu_{1}$ is not detected at $D_{1^{\prime}}$, but has not yet been detected at $D_{1}, \nu_{2}$ may be forced into a well-defined polarization state, or not, depending on the capability of NR detections to induce a reduction of the state vector.

where $|a\rangle\left(\left|a_{\perp}\right\rangle\right)$ represents a linear polarization state parallel (perpendicular) to a. $\nu_{1}$ and $\nu_{2}$ are sent in opposite directions: $\nu_{1}$ impinges on a two-channel polarizer oriented parallel to a, and $\nu_{2}$ (after following a detour) on a twochannel polarizer oriented parallel to $\mathbf{b}$. Photons $\nu_{1}$ that are transmitted have to follow a detour, before impinging on detector $D_{1}$. Photons $\nu_{1}$ that are reflected impinge on detector $D_{1^{\prime}}$. Photons $\nu_{2}$ that are transmitted (reflected) impinge on detector $D_{2}\left(D_{2^{\prime}}\right)$. The distances are such that, independently of the Lorentz frame we use to describe the experiment, $\nu_{1}$ is always detected (or not detected) at detector $D_{1^{\prime}}$ before $\nu_{2}$ is detected at $D_{2}$ or $D_{2^{\prime}}$, and $\nu_{2}$ is always detected at $D_{2}$ or $D_{2^{\prime}}$ before $\nu_{1}$ is detected at $D_{1}$. Therefore, the detection of $\nu_{1}$ at $D_{1}$ cannot force $\nu_{2}$ into a well-defined polarization state; similarly, the detection of $\nu_{2}$ at $D_{2}$ or $D_{2^{\prime}}$ cannot force $\nu_{1}$ into a well-defined polarization state, since $\nu_{1}$ has already either been or not been detected at $D_{1^{\prime}}$; the only possibility is the detection (or non-detection) of $\nu_{1}$ at $D_{1}$, forcing $\nu_{2}$.

If, as generally believed, NR detections are capable of inducing the reduction of the state vector, we must have

$$
p\left(a_{\perp}, b\right)=p\left(a, b_{\perp}\right)=\frac{1}{2} \sin ^{2}(a, b)
$$

and

$$
p\left(a_{\perp}, b_{\perp}\right)=p(a, b)=\frac{1}{2} \cos ^{2}(a, b)
$$


where $p\left(a_{\perp}, b\right)$ is the probability of detecting $\nu_{1}$ in a polarization state perpendicular to $\mathbf{a}$ and $\nu_{2}$ in a polarization state parallel to $\mathbf{b}$, and so on. Please, note, for instance, that we can write $p\left(a_{\perp}, b\right)=p\left(a_{\perp}\right) p\left(b \mid a_{\perp}\right)$, where $p\left(a_{\perp}\right)=1 / 2$ is the probability of $\nu_{1}$ being detected at $D_{1^{\prime}}$, and $p\left(b \mid a_{\perp}\right)=\sin ^{2}(a, b)$ is the probability of $\nu_{2}$ being detected at $D_{2}$ when $\nu_{1}$ has been detected at $D_{1^{\prime}}$, forcing $\nu_{2}$ into a state perpendicular to a. Similarly, assuming that NR-detections are capable of inducing the reduction of the state vector, we can write, for instance, $p\left(a, b_{\perp}\right)=p(a) p\left(b_{\perp} \mid a\right)$, where $p(a)=1 / 2$ is the probability of $\nu_{1}$ not being detected at $D_{1^{\prime}}$, and $p\left(b_{\perp} \mid a\right)=\sin ^{2}(a, b)$ is the probability of $\nu_{2}$ being detected at $D_{2^{\prime}}$ when $\nu_{1}$ has not been detected at $D_{1^{\prime}}$, but has not been detected at $D_{1}$ yet, even so forcing $\nu_{2}$ into a state parallel to a. On the other hand, if NR detections are incapable of inducing the reduction of the state vector, we must have

$$
\begin{gathered}
p^{\prime}\left(a_{\perp}, b\right)=p\left(a_{\perp}, b\right)=\frac{1}{2} \sin ^{2}(a, b), \\
p^{\prime}\left(a_{\perp}, b_{\perp}\right)=p\left(a_{\perp}, b_{\perp}\right)=\frac{1}{2} \cos ^{2}(a, b), \\
p^{\prime}(a, b)=\frac{1}{2} \alpha(a, b)
\end{gathered}
$$

and

$$
p^{\prime}\left(a, b_{\perp}\right)=\frac{1}{2}[1-\alpha(a, b)],
$$

where, in general, $\alpha(a, b)=p^{\prime}(b \mid a) \neq p(b \mid a)=\cos ^{2}(a, b)$, since, when $\nu_{1}$ is not detected at $D_{1^{\prime}}, \nu_{2}$ (which, in this case, is detected before $\nu_{1}$ ) is not forced into a polarization state parallel to a [17]. Naturally, when $\nu_{1}$ is detected at $D_{1^{\prime}}$, $\nu_{2}$ is forced into a polarization state perpendicular to $\mathbf{a}$, and the usual quantum mechanical results are obtained (eq. (4) and (5)). It is interesting to observe that

$$
p^{\prime}(b)=p^{\prime}(a, b)+p^{\prime}\left(a_{\perp}, b\right)=\frac{1}{2}\left[\alpha(a, b)+\sin ^{2}(a, b)\right]
$$

and

$$
p^{\prime}\left(b_{\perp}\right)=p^{\prime}\left(a, b_{\perp}\right)+p^{\prime}\left(a_{\perp}, b_{\perp}\right)=\frac{1}{2}\left[1-\alpha(a, b)+\cos ^{2}(a, b)\right] .
$$

Therefore, in this hybrid situation, in which $\nu_{2}$ is forced into a well-defined polarization state whenever $\nu_{1}$ is detected at $D_{1^{\prime}}$, and no forcing occurs if $\nu_{1}$ is not detected at $D_{1^{\prime}}$, we may have $p^{\prime}(b) \neq p^{\prime}\left(b_{\perp}\right)$.

To determine the disagreement between the NR-detection reduction of the state vector (NR-detection collapse, for short) and the no-NR-detection collapse approaches, I will introduce a local model [6]. This artifice needs a brief explanation. I am assuming that whenever $\nu_{1}$ is not detected at $D_{1^{\prime}}$, but has not yet been detected at $D_{1}$, nothing happens to $\nu_{2}$. In this case, supposing that $\nu_{2}$ remains unpolarized, we simply must have $\alpha(a, b)=1 / 2$. However, for the sake of completeness, I will also contemplate the possibility of having a local hidden variable (LHV) connection between the entangled photons, that is: $\alpha(a, b) \equiv \alpha(a, b ; \lambda)$, where $\lambda$ stands for all possible LHV. In other words, there might be some properties previously shared by the particles. In order to have space-like events, we can remove the detours in the experiment represented in Fig.1; however, the results would not change if we considered time-like events 
instead, since the correlations are supposed to be determined only by the previously shared properties. Using (7) and (6), the probabilities will be given by

$$
p_{L}\left(a_{\perp}, b\right)=p_{L}\left(a, b_{\perp}\right)=p^{\prime}\left(a, b_{\perp}\right)=\frac{1}{2}[1-\alpha(a, b)]
$$

and

$$
p_{L}\left(a_{\perp}, b_{\perp}\right)=p_{L}(a, b)=p^{\prime}(a, b)=\frac{1}{2} \alpha(a, b) .
$$

Hence, the correlation function will be

$$
\begin{gathered}
E_{L}(a, b)=\frac{p_{L}(a, b)-p_{L}\left(a, b_{\perp}\right)-p_{L}\left(a_{\perp}, b\right)+p_{L}\left(a_{\perp}, b_{\perp}\right)}{p_{L}(a, b)+p_{L}\left(a, b_{\perp}\right)+p_{L}\left(a_{\perp}, b\right)+p_{L}\left(a_{\perp}, b_{\perp}\right)} \\
=2 \alpha(a, b)-1 .
\end{gathered}
$$

Using the CHSHB inequality $[4,16]$

$$
\left|E_{L}(a, b)-E_{L}\left(a, b^{\prime}\right)+E_{L}\left(a^{\prime}, b\right)+E_{L}\left(a^{\prime}, b^{\prime}\right)\right| \leq 2,
$$

choosing angle $(a, b)=\operatorname{angle}\left(a^{\prime}, b\right)=\operatorname{angle}\left(a^{\prime}, b^{\prime}\right)=\frac{1}{3} \operatorname{angle}\left(a, b^{\prime}\right)=22.5^{\circ}$, and taking into account that $E_{L}\left(67.5^{\circ}\right)=-E_{L}\left(22.5^{\circ}\right)$, we obtain

$$
4 E_{L}\left(22.5^{\circ}\right) \leq 2
$$

which, using (12), leads to

$$
\alpha\left(22.5^{\circ}\right) \leq \frac{3}{4}
$$

Using (8) and (9) we obtain

$$
p^{\prime}\left(b_{\perp}\right)-p^{\prime}(b)=\frac{1}{2}-\alpha+\frac{1}{2} \cos 45^{\circ},
$$

which, using (15), leads to

$$
p^{\prime}\left(b_{\perp}\right)-p^{\prime}(b) \geq \frac{\sqrt{2}-1}{4} \approx 0.1,
$$

in disagreement with the quantum mechanical prediction $p\left(b_{\perp}\right)-p(b)=0$. Naturally, if there is no classical correlation, $\alpha=$ const. $=1 / 2, p^{\prime}(a, b)=$ $p^{\prime}\left(a, b_{\perp}\right)=$ const. $=1 / 4$, and instead of $(17)$ we will have $p^{\prime}\left(b_{\perp}\right)-p^{\prime}(b) \approx 0.35$. But, in this case, the greatest disagreement is obtained choosing $\mathbf{a}=\mathbf{b}$, which, using (9) and (8), leads to $p^{\prime}\left(b_{\perp}\right)=3 / 4$ and $p^{\prime}(b)=1 / 4$, and, using (7), to $p^{\prime}\left(b, b_{\perp}\right)=1 / 4 \neq p\left(b, b_{\perp}\right)=0$.

\section{The Real Situation}

In a real situation (assuming, for purposes of simplification, that all detectors have the same efficiency, and that the two polarizers are identical), instead of (2) and (3), we have [16]

$$
p(a, b)=p\left(a_{\perp}, b_{\perp}\right)=\frac{1}{4} \eta^{2} f g\left[T_{+}^{2}+F T_{-}^{2} \cos 2(a, b)\right]
$$

and

$$
p\left(a, b_{\perp}\right)=p\left(a_{\perp}, b\right)=\frac{1}{4} \eta^{2} f g\left[T_{+}^{2}-F T_{-}^{2} \cos 2(a, b)\right],
$$


where $\eta$ is the detectors' efficiency; $f$ is the probability of the first photon being collected; $g$ is the probability of the second photon being collected when the first has been collected; $T_{ \pm}=T_{\|} \pm T_{\perp}$, where $T_{\|}\left(T_{\perp}\right)$ is the transmission coefficient for light polarized parallel (perpendicular) to the polarizer's orientation; and $F$ indicates the amount of correlation between the photons. Actually, unlike what has been done in section II, the best procedure (from a practical point of view) is to consider only the coincident detections in which $D_{1}$ clicks, since when $D_{1^{\prime}}$ clicks no NR detection occurs. From (18) and (19) we see that

$$
p_{c}(a)=p(a, b)+p\left(a, b_{\perp}\right)=\frac{1}{2} \eta^{2} f g T_{+}^{2},
$$

where the subscript $c$ indicates that we are only considering situations in which $\nu_{1}$ and $\nu_{2}$ are both detected (coincident detections). Therefore,

$$
p(b \mid a)=\frac{p(a, b)}{p_{c}(a)}=\frac{1}{2}\left[1+F \frac{T_{-}^{2}}{T_{+}^{2}} \cos 2(a, b)\right]
$$

and

$$
p\left(b_{\perp} \mid a\right)=\frac{p\left(a, b_{\perp}\right)}{p_{c}(a)}=\frac{1}{2}\left[1-F \frac{T_{-}^{2}}{T_{+}^{2}} \cos 2(a, b)\right],
$$

where $p(b \mid a)\left[p\left(b_{\perp} \mid a\right)\right]$ is the probability of $\nu_{2}$ being detected at $D_{2}\left[D_{2^{\prime}}\right]$ when $\nu_{1}$ is detected at $D_{1}$, and (18), (19), and (20) have been used. Using (21) and (22) we obtain

$$
p(b \mid a)-p\left(b_{\perp} \mid a\right)=F \frac{T_{-}^{2}}{T_{+}^{2}} \cos 2(a, b),
$$

which can be written, using only directly observable quantities, as

$$
\frac{N(a, b)-N\left(a, b_{\perp}\right)}{N(a, b)+N\left(a, b_{\perp}\right)}=F \frac{T_{-}^{2}}{T_{+}^{2}} \cos 2(a, b),
$$

where $N(a, b)\left[N\left(a, b_{\perp}\right)\right]$ is the number of coincident detections at $D_{1}$ and $D_{2}\left[D_{2^{\prime}}\right]$. Hence, using the data from the experiment by Aspect, Grangier, and Roger [16] $\left(T_{\|} \approx 0.950, T_{\perp} \approx 0.007\right.$, and $\left.F \approx 0.984\right)$, we see that

$$
\frac{N(a, b)-N\left(a, b_{\perp}\right)}{N(a, b)+N\left(a, b_{\perp}\right)} \approx 0.696, \quad\left[\operatorname{angle}(a, b)=22.5^{\circ}\right] .
$$

On the other hand, in a real situation, if no NR-detection collapse occurs, instead of (6) and (7) we must have

$$
p^{\prime}(a, b)=\frac{1}{4} \eta^{2} f g\left[T_{+}^{2}+\beta(a, b)\right]
$$

and

$$
p^{\prime}\left(a, b_{\perp}\right)=\frac{1}{4} \eta^{2} f g\left[T_{+}^{2}-\beta(a, b)\right],
$$

where, in general, $\beta(a, b) \neq F T_{-}^{2} \cos 2(a, b)$. Introducing a local model satisfying the conditions

$$
p_{L}(a, b)=p_{L}\left(a_{\perp}, b_{\perp}\right)=p^{\prime}(a, b)
$$

and

$$
p_{L}\left(a, b_{\perp}\right)=p_{L}\left(a_{\perp}, b\right)=p^{\prime}\left(a, b_{\perp}\right),
$$


instead of (12) we obtain

$$
E_{L}(a, b)=\frac{\beta(a, b)}{T_{+}^{2}}
$$

which, using (13), leads to

$$
\frac{\beta(a, b)}{T_{+}^{2}} \leq \frac{1}{2}, \quad\left[\operatorname{angle}(a, b)=22.5^{\circ}\right] .
$$

From (26) and (27) we have

$$
p_{c}^{\prime}(a)=p^{\prime}(a, b)+p^{\prime}\left(a, b_{\perp}\right)=\frac{1}{2} \eta^{2} f g T_{+}^{2} .
$$

Hence,

$$
p^{\prime}(b \mid a)=\frac{p^{\prime}(a, b)}{p_{c}^{\prime}(a)}=\frac{1}{2}\left[1+\frac{\beta(a, b)}{T_{+}^{2}}\right]
$$

and

$$
p^{\prime}\left(b_{\perp} \mid a\right)=\frac{p^{\prime}\left(a, b_{\perp}\right)}{p_{c}^{\prime}(a)}=\frac{1}{2}\left[1-\frac{\beta(a, b)}{T_{+}^{2}}\right],
$$

where (26), (27), and (32) have been used. Therefore, using (31), we can write

$$
p^{\prime}(b \mid a) \leq \frac{1}{2}\left(1+\frac{1}{2}\right)
$$

and

$$
p^{\prime}\left(b_{\perp} \mid a\right) \geq \frac{1}{2}\left(1-\frac{1}{2}\right)
$$

which leads to

$$
p^{\prime}(b \mid a)-p^{\prime}\left(b_{\perp} \mid a\right) \leq \frac{1}{2}
$$

and to

$$
\frac{N^{\prime}(a, b)-N^{\prime}\left(a, b_{\perp}\right)}{N^{\prime}(a, b)+N^{\prime}\left(a, b_{\perp}\right)} \leq 0.5, \quad\left[\operatorname{angle}(a, b)=22.5^{\circ}\right]
$$

in strong disagreement with (25). Assuming, in Fig.1, the distance from $S$ to the first polarizer as being equal to 1 meter, we can easily see that the lengths of the detours for $\nu_{2}$ and $\nu_{1}$, respectively, can be of approximately 2 and $4 \mathrm{~m}$, which can easily be accomplished using optical fibers.

\section{Discussion}

As emphasized at the beginning of this paper, NR-detection collapse is far from being a trivial fact, and as such deserves to be experimentally investigated. As already stressed in ref. 5 and 6 , an interesting result is that, if NR-detection collapse does not occur, then FTL communication becomes possible, at least in principle, provided we are able to establish which photon of an entangled pair is really detected first [18]. For instance, by monitoring the number of detections on the right-hand side of the experimental apparatus (Fig.1), it would be possible to know whether detector $D_{1}$ on the left-hand side has been "removed" (that is, if a detour has been introduced) or not [19], since it follows from (17) that $p^{\prime}\left(b_{\perp}\right) \neq p^{\prime}(b)$. This is in agreement with Svetlichny's arguments [20] supporting 
the standpoint according to which a causal theory (i.e. without superluminal communication) implies formal state collapse. Although, at first sight, FTL communication seems inconsistent with special relativity, things may not be so simple. As shown in the appendix (and surprising as it may sound), no causal paradox necessarily arises from superluminal signaling, even maintaining the Lorentz transformations.

The second possible result is that, as expected, NR-detection collapse occurs. In this case, from an ontological standpoint (namely, assuming that the collapse involves a change in the physical properties of the system), there seem to be two alternatives [21]: (a) adopting a pilot wave interpretation a la de Broglie-Bohm, we can assume that $\nu_{2}$ is forced into a well-defined polarization state when $\nu_{1}$ is split in the polarizer into a "full" wave and an "empty" wave [22]; (b) ascribing an objective meaning (or substance, so to speak) to the probability amplitude [23], we have to treat $\mathrm{O}$ and NR detections on the same footing; that is, each time an $\mathrm{O}$ detection (or more generally, a photon absorption) fails to occur in one branch of the experiment, the probability amplitudes associated to the other branches are altered (as a consequence of the changes experienced by the physical system), and automatically adjusted: instead of photon absorption, we have the absorption (actually, the redistribution) of a probability amplitude, which corresponds to an NR detection.

The above remarks raise the question about the possibility of quantum mechanics being superseded. The most immediate thought that comes to mind is of a new theory that keeps many essential features of the "old" one. However, Bohr's model of the atom, in which accelerated charges do not necessarily radiate, is a good example of the fact that it is not always wise to become too attached to the prevailing views, even though these views will eventually play an important role in a more elaborate formulation of the new theory [24]. NR detections (if capable of inducing a collapse of the wavefunction, as expected) strongly suggest that no amplification is involved in the reduction of the state vector; in this case, adopting an ontological point of view [12], we may conjecture that at a more fundamental level than that ruled by quantum mechanics some as yet unknown processes take place which are responsible for the so called "actualization of potentialities".

\section{Conclusion}

In this paper it has been assumed that the ordinary $(\mathrm{O})$ detection of a photon of a polarization entangled pair forces its twin into a well-defined polarization state. On the other hand, the consequences of the conjecture that this might not be true for null-result (NR) detections have been investigated. More specifically, it has been pointed out that no noncontroversial collapse inducing NR experiment has been performed so far, and a suggestion to remedy this situation has been presented. Naturally, if the proposed experiment was conducted and had a surprising result (i.e. if it was found that NR-detection does not reduce the state vector), this would have significance for ordinary quantum theory, and, furthermore, it would allow us to infer an important thing about nature, viz. that, as stressed in the paper, superluminal communication would be possible in principle. As shown in the Appendix, this does not necessarily imply a causal paradox or the abandonment of Lorentz transformations. Actually, I would be very surprised if it was found that NR-detections do not reduce the state vec- 
tor. However, I also think that we can only be certain about this point after a conclusive experiment has been performed. As stressed in the previous section, whatever the outcome of the experiment that is being proposed, it will have important consequences for the foundations of QM.

\section{Appendix: Superluminal Signaling Without Causal Paradox}

The prevalent opinion is that the idea of faster-than-light (FTL) communication leads to causal paradoxes. Although, in principle, the introduction of a privileged frame would circumvent this problem, it is not immediately obvious how to conciliate FTL communication with the Lorentz transformations. Here it is shown-and, as far as I know, this is a new result-how to do this by breaking the Lorentz symmetry; that is, although the Lorentz transformations remain valid, the equivalence between passive and active transformations is violated when superluminal communication is considered.

Let us suppose that behind EPR correlations there is an FTL interaction that can be used for superluminal communication, an idea entertained by Bohm [8]. As we will see, this does not necessarily lead to causal paradoxes, provided that we assume the existence of a preferred frame (an aether, as imagined by Bell [19]) in which the speed of the FTL interaction is a constant, that is, it is always the same, independently of the motion of the "source" (or, equally, of the reference frame in which the experiment is being performed). Let us consider a pair of reference frames, $\mathbf{S}$ and $\mathbf{S}^{\prime}$, in the standard configuration, where $\mathbf{S}$ is the preferred frame and $\mathbf{S}^{\prime}$ moves with velocity $v<c$ along the $x$ axis. Assuming that the Lorentz transformations

$$
\begin{aligned}
x^{\prime} & =\gamma(x-v t), \\
t^{\prime} & =\gamma\left(t-\frac{v}{c^{2}} x\right), \\
x & =\gamma\left(x^{\prime}+v t^{\prime}\right),
\end{aligned}
$$

and

$$
t=\gamma\left(t^{\prime}+\frac{v}{c^{2}} x^{\prime}\right),
$$

connect the $\mathbf{S}$ and $\mathbf{S}^{\prime}$ coordinates (with $\gamma=1 / \sqrt{1-v^{2} / c^{2}}$ ), we derive

$$
u^{\prime}=\frac{u-v}{1-\frac{v u}{c^{2}}}
$$

and

$$
u=\frac{u^{\prime}+v}{1+\frac{v u^{\prime}}{c^{2}}}
$$

for the velocities [25].

Let us, initially, see how the causal paradox arises in special relativity (in which there is no preferred frame and $\mathbf{S}$ and $\mathbf{S}^{\prime}$ are equivalent). Let the positive quantity $\bar{u}>c$ represent the superluminal signal speed in $\mathbf{S}$. From $(a .5)$, we see that if $u=\bar{u}$, we can choose $v$ so as to have $v \bar{u} / c^{2}>1$, which leads to $u^{\prime}<0$ (with $\left|u^{\prime}\right|>c$ but $\neq \bar{u}$ ). Therefore, in $\mathbf{S}^{\prime}$ the signal propagates backwards. Similarly, from $(a .6)$ we see that, if $u^{\prime}=-\bar{u}$, we can choose a $v$ that leads to $u>0$ (with $u>c$ and $\neq \bar{u}$ ). That is, in $\mathbf{S}$ the direction of propagation of the 
signal is reversed. It is this change of direction when we go from $\mathbf{S}$ to $\mathbf{S}^{\prime}$, and then from $\mathbf{S}^{\prime}$ to $\mathbf{S}$, that is at the origin of the causal paradox. To see this, let us consider a superluminal signal emitted from $x_{0}=0$, at instant $t_{0}=0$, and reaching $x_{1}>0$ at instant $t_{1}$ given by

$$
t_{1}=\frac{x_{1}}{\bar{u}}
$$

in $\mathbf{S}$. In $\mathbf{S}^{\prime}$, the signal is transmitted from $x_{0}^{\prime}=0$, at instant $t_{0}^{\prime}=0$, reaching $x_{1}$ at instant

$$
t_{1}^{\prime}=\gamma\left(t_{1}-\frac{v}{c^{2}} x_{1}\right)=\gamma\left(1-\frac{v \bar{u}}{c^{2}}\right) \frac{x_{1}}{\bar{u}}
$$

according to $(a .2)$ and $(a .7)$. We see that $v \bar{u} / c^{2}>1 \rightarrow t_{1}^{\prime}<0$. Therefore, in $\mathbf{S}^{\prime}$ the signal reaches $x_{1}$ before it is sent from $x_{0}$ (actually, the signal is seen to propagate from $x_{1}$ to $x_{0}$ ). But this does not yet represent a paradox, since no contradiction ( $A$ and $\neg A$, for instance) is occurring. Let us then determine the point $x_{1}^{\prime}$ in $\mathbf{S}^{\prime}$ that coincides with $x_{1}$ at the instant at which the signal arrives. Using (a.1) and (a.7), we obtain

$$
x_{1}^{\prime}=\gamma\left(x_{1}-v \frac{x_{1}}{\bar{u}}\right)=\gamma\left(1-\frac{v}{\bar{u}}\right) x_{1} .
$$

An observer at $x_{1}^{\prime}$ can then send a return signal with $u^{\prime}=-\bar{u}$ that will take the time of

$$
\delta t^{\prime}=\frac{x_{1}^{\prime}}{\bar{u}}=\gamma\left(1-\frac{v}{\bar{u}}\right) \frac{x_{1}}{\bar{u}}
$$

to arrive at $x_{0}^{\prime}$. This can lead to a paradox if

$$
t_{1}^{\prime}+\delta t^{\prime}<0
$$

that is, if the return signal reaches the origin of $\mathbf{S}^{\prime}$ before $t_{0}^{\prime}$, namely before the first signal has been sent. This enables an observer in this region, after receiving the return signal, to inform another observer, at the origin of $\mathbf{S}$, not to send the signal. As a consequence, if the signal is sent, it is possible to send a return signal to impede the emission of the signal. That is, the signal would be sent and not sent at the same time! Let us see the condition $v$ would have to fulfil. From (a.11), (a.10), and (a.8), we obtain

$$
\gamma\left(1-\frac{v \bar{u}}{c^{2}}\right) \frac{x_{1}}{\bar{u}}+\gamma\left(1-\frac{v}{\bar{u}}\right) \frac{x_{1}}{\bar{u}}<0,
$$

which leads to

$$
v>\frac{2 \bar{u}}{1+\frac{\bar{u}^{2}}{c^{2}}} .
$$

Since the right-hand side of (a.13) is always smaller than $c$, it is always possible to find a $v$ that satisfies the above condition; therefore, we would indeed have a paradox.

Now let us see how the existence of a preferred frame in which the superluminal speed is a constant does not lead to a causal paradox. Instead of (a.10), we have

$$
\delta t^{\prime}=\frac{x_{1}^{\prime}}{-\bar{u}^{\prime}}=-\gamma\left(1-\frac{v}{\bar{u}}\right) \frac{x_{1}}{\bar{u}^{\prime}},
$$


where the velocity of the return signal (using $(a .5)$ ) is

$$
\bar{u}^{\prime}=\frac{-\bar{u}-v}{1+\frac{v \bar{u}}{c^{2}}} .
$$

The condition to have a causal paradox is then

$$
\gamma\left(1-\frac{v \bar{u}}{c^{2}}\right) \frac{x_{1}}{\bar{u}}-\gamma\left(1-\frac{v}{\bar{u}}\right) \frac{x_{1}}{\bar{u}^{\prime}}<0
$$

where (a.14), (a.11), and (a.8) have been used. From (a.15) and (a.16) we obtain

$$
v>c
$$

which contradicts our initial assumption that the velocity of reference frame $\mathbf{S}^{\prime}$ is slower than the velocity of light. As a consequence, there can be no causal paradox.

Please note that the superluminal interaction that is being considered here breaks the Lorentz symmetry, since the active transformation that would correspond to the passive transformation does not exist (we have a similar situation in the case of the violation of parity, in which some mirror-reflected phenomena have no counterpart in the real world); that is, to describe the FTL experiment from a frame that moves with velocity $\mathbf{- v}$ relative to the preferred frame (passive transformation) is not the same as to stay in the preferred frame and describe an FTL experiment in which the experimental apparatus moves with velocity $\mathbf{v}$ (active transformation).

Acknowledgment: I thank Paulo Henrique Souto Ribeiro for helpful conversations.

\section{References}

[1] The terms "null" measurement, "null-result" measurement, and "negativeresult" measurement are also used.

[2] Quantum Theory and Measurement, edited by J. A. Wheeler and W. H. Zurek (Princeton University Press, 1983).

[3] Home and Whitaker, in D. Home and M. A. B. Whitaker, J. Phys. A: Math. Gen. 25, 2387 (1992), discuss different kinds of negative-result experiments, and argue that they can be explained without the need to invoke the collapse of the wave function.

[4] J. S. Bell, Speakable and Unspeakable in Quantum Mechanics (Cambridge University Press, 1989), collected papers on foundations of quantum mechanics; J. F. Clauser and A. Shimony, Rep. Prog. Phys. 41, 1881 (1978), A. Aspect, Thèse d'Etat (Orsay, 1983); Y. H. Shi and C. O. Alley, Phys. Rev. Lett. 61, 2921 (1998); G. Weihs, T. Jennewein, C. Simon, H. Weinfurter, and A. Zeilinger, Phys. Rev. Lett. 81, 5039 (1998); T. B. Pittman and J. D. Franson, Phys. Rev. Lett. 90, 240401 (2003). 
[5] L. C. Ryff, Phys. Lett. A 170, 259 (1992), in which the term "indirect detection" is used. But, in general, indirect detection involves the inference of the existence of something (e.g., elementary particles, dark matter, via Weakly Interacting Massive Particles (WIMPs), et cetera) from the detection of some other thing.

[6] L. C. Ryff, in Mysteries, Puzzles, and Paradoxes in Quantum Mechanics, edited by R. Bonifacio (American Institute of Physics, 1999); L. C. Ryff and C. H. Monken, Quantum Semiclass. Opt. 1, 345 (1999), in which the term "interaction-free measurement" is used. However, this term has become linked to the kind of experiment discussed in A. Elitzur and L. Vaidman, Found. Phys. 23, 987 (1993), which, as will become evident, is actually quite different in spirit from the kind of experiment which is being discussed here. In the Elitzur-Vaidman proposal, whenever a photon is detected in the "forbidden" output port, it is possible to infer the presence of a light absorbing object in one of the arms of a Mach-Zehnder interferometer. Here, whenever a detector does not click, it is possible to infer the path followed by a photon.

[7] A. Einstein, B. Podolsky, and N. Rosen, Phys. Rev. 47, 777 (1935); D. Bohm, Quantum Theory (Prentice-Hall, 1951).

[8] In an interview in The Ghost in the Atom, eds P. C. W. Davies and J. R. Brown (Cambridge University Press, 1989), Bohm explicitly entertains the idea of FTL signaling.

[9] P. G. Kwiat, K. Mattle, H. Weinfurter, A. Zeilinger, A. V. Sergienko, and Y. Shih, Phys. Rev. Lett. 75, 4337 (1995); P. G. Kwiat, E. Waks, A. G. White, I. Appelbaum, and P. H. Eberhard, Phys. Rev. A 60, R773 (1999).

[10] It may sound strange to consider the lack of detection as an event; however, taking into account the context, there seems to be no reason for any confusion.

[11] Actually, the transmitted and reflected probability amplitudes can be recombined by means of a Mach-Zehnder interferometer, and the detectors can be placed at the interferometer output ports, so that interference can be observed. As a result, we don't, strictly speaking, have a classical situation. However, as has been stressed, it admits, in principle, a simple interpretation based on the de Broglie-Bohm pilot-wave approach (D. Bohm and J. B. Hiley, The Undivided Universe: An Ontological Interpretation of Quantum Theory (Routledge, 1993)), in which a wave with a photon ("full wave") follows one arm of the interferometer and a wave without a photon ("empty wave") follows the other (F. Selleri, in The Wave Particle Dualism, edited by S. Diner et al (Kluwer Academic Publishers, 1984); T. Norsen, quantph/0611034 (2006)). The possibility of introducing photon trajectories has also been investigated, for example, in P. Ghose, A. S. Majundar, S. Guha, and J. Sau, Phys. Lett. A 290, 205 (2001), and A. S. Sanz, M. Davidović, M. Božić, and S. Miret-Artés, Annals of Phys. 325, 763 (2010), and references there in. As a consequence, for this kind of experiment, the consideration of time-like events (by introducing a detour on the photon path leading to the distant detector, for example) would not change it into an indisputable 
NR-detection experiment. Similarly, by simply changing the position of the detectors, placing the first distant from and the second close to the beam splitter, this does not improve the situation.

[12] Here, "objective" change of probability is to be understood as a change in probability as a consequence of a change in the physical properties of the system. A "subjective" change of probability is associated to a Bayesian point of view, in which the probability is automatically updated when our knowledge changes. According to Polkinghorne, "One must acknowledge that a true case of action at a distance is involved, and not merely some gain in additional knowledge. Putting in a learned language, the EPR effect is ontological and not simply epistemological.", J. Polkinghorne, Quantum Theory: A Very Short Introduction (Oxford University Press, 2002), p. 80 (a very lucid and concise explanation of the foundations of quantum mechanics). T. Maudlin, Quantum Non-Locality and Relativity (Blackwell, 2002 ) is also a good read.

[13] M. Renninger, Ztschr. Phys. 158, 417 (1960). Renninger has been considered to be the first to draw attention to null measurements (as in H. Paul, Introduction to Quantum Theory (Cambridge University Press, 2008), page 59); however, in an interesting article, M. A. B. Whitaker, Prog. Quant. Electron. 24, 1 (2000), discusses the gedanken experiment suggested in a precursory paper by P. S. Epstein, Am. J. Phys. 13, 127 (1945).

[14] R. H. Dicke, Am. J. Phys. 49, 925 (1981), in which-as far as I know-the term "interaction-free" measurement has been introduced.

[15] Since some loopholes still remain, it follows that, strictly speaking, we cannot affirm that we know from the empirical violations of Bell's inequalities that the successful detection of a particle can collapse the wave function and thus force the other distant particle of the pair into a well-defined state. In my opinion, from a Popperian standpoint, the only thing we can say is that the quantum mechanical predictions have been corroborated by experiment. Exactly when and where this "forcing" (which, in principle, can be accepted even for those who reject the idea of collapse) can be considered accomplished is a question still open to debate. It can be conjectured, based on the pilot-wave interpretation, for instance, that it takes place whenever the first photon is split at the two-channel polarizer into a wave with a photon and a wave without a photon. However, according to Bohm and Hiley (ref. 11), in the case of boson fields we should give up the notion of particle-and, naturally, also of pilot wave-and consider the field variables themselves as fundamental ontological entities. But it does not seem clear in this approach how to deal with NR-detections. As acknowledged by P. R. Holland on page 481 of his book entitled The Quantum Theory of Motion: An Account of the de Broglie-Bohm Causal Interpretation of Quantum Mechanics (Cambridge University Press, 1995), a detailed treatment of the optical experiments testing Bell's inequalities has not yet been given in the deBroglie-Bohm causal interpretation. On the other hand, in the theories based on the idea of spontaneous collapse (A. Bassi and G. C. Ghirardi, Phys. Rep. 379, 257 (2003); G.Ghirardi, http://plato.stanford.edu/archives/win2011/entries/qm-collapse/) it 
seems that the detector must be, during an exceedingly short time, in a superposition of fired and not fired states. There are also other possible interpretations, based on the decoherence approach ( O. Pessoa Jr, Synthese, 113 (1998); S. L. Adler, Stud. Hist. Philos. Mod. Phys., 34, 135 (2003); M. Schlosshauer, Rev. Mod. Phys. 76, 1267 (2004); G. Bacciagaluppi, http://plato.stanford.edu/archives/fall2008/entries/qmdecoherence/), on many-worlds (A. Kent, arxiv:gr-qc/9703089; L. Vaidman, $\quad$ http://plato.stanford.edu/archives/fall2008/entries/qmmanyworlds/), and so forth. I think that to discuss all these alternatives would lead me astray from the main point, namely, that independently of the explanation we prefer, NR detections have not been satisfactorily tested.

[16] A. Aspect, P. Grangier, and G. Roger, Phys. Rev. Lett. 49, 91 (1982).

[17] To avoid any conceptual confusion, it is important to distinguish between the terms collapsing and forcing in the present context. Although $\nu_{2}$ is not forced into a well-defined polarization state (and, therefore, no "ordinary" reduction or collapse of the state vector occurs), $\nu_{1}$ can no longer be detected at $D_{1^{\prime}}$, as a consequence, equation (1) is not valid. More specifically, $\nu_{1}$ can no longer be detected in state $\left|a_{\perp}\right\rangle$, otherwise, we would have the strange situation in which, by introducing a second polarizer after the first one with the same orientation, $\nu_{1}$ could be transmitted at the first polarizer and reflected at the second. Whether this should be considered a sort of collapse, I prefer, by the moment, to leave it as an open question. It is also important to notice that, when writing the probabilities $p(b \mid a)$ and $p^{\prime}(b \mid a)$, we are assuming that whenever $\nu_{1}$ is not detected at $D_{1^{\prime}}$ it will necessarily be detected at $D_{1}$. Otherwise, we would have more detections at $D_{1}$ than at $D_{1}$.

[18] V. Scarani and N. Gisin, Braz. J. Phys. 35, 328 (2005), and Phys. Lett. A 295, 167 (2002), have shown that the assumption of finite speed superluminal interaction leads, assuming that the correlations are purely nonlocal, and for more than two entangled particles, to FTL signalling. The same subject has been approached, from a different standpoint in L. C. Ryff, arXiv: quant-ph/0903.1076. An interesting discussion of this theme is in N. Gisin, arXiv:quant-ph/1011.3440. For more recent and general results, see J-D. Bancal et al., Nature Physics 8, 867 (2012) and T. J. Barnea et al., Phys. Rev. A 88, 022123 (2013).

[19] Actually, to have FTL communication we have to consider space-like events (no detours in Fig. 1) and all $\nu_{2}$ that are detected, for instance, independently of $\nu_{1}$ also being detected or not (no classical communication channel to inform on that). We also have to implicitly assume that there is a preferred frame in which one of the photons $\left(\nu_{1}\right.$, in this example) is really detected first. Arguments in favor of a preferred frame have been presented by John Bell in "How to teach special relativity," in J. S. Bell, ref. 4, and in an interview in The Ghost in the Atom, eds P. C. W. Davies and J. R. Brown (Cambridge University Press, 1989). Bohm and Hiley also advocate a preferred frame, in D. Bohm and J. B. Hiley, ref. 11. The possibility of introducing and identifying a preferred frame is examined in P. Caban and 
J. Rembieliński, Phys. Rev. A 59, 4187 (1999), in J. Rembieliński and K. A. Smoliński, Phys. Rev. A 66, 052114 (2002), and more recently in J. Rembieliński and K. A. Smoliński, EPL 88, 10005 (2009).

[20] G. Svetlichny, Found. Phys. 33, 641 (2003). The use of the no signaling condition as an axiom to build the fundamental structure of quantum mechanics has also been discussed in: G. Svetlichny, Found. Physics 28, 131 (1998); G. Svetlichny, Found. Physics 30, 1819 (2000); and C. Simon, V. Bužek, and N. Gisin, Phys. Rev. Lett. 87, 170405 (2001).

[21] Although this paper can be considered a contribution to the important "psi-ontic vs. psi-epistemic" issue debate (C. A. Fuchs and R. Schack, arXiv:quant-ph/1301.3274, and references therein), it is not intended to settle it. My personal reasons to prefer an ontic point of view are briefly expounded in L. C. Ryff, arXiv:quant-ph/1308.5690. Retrocausal interpretations are also interesting (J. G. Cramer, Found. Phys. Lett. 19, 63 (2006), and references therein), but, to me, it is not yet clear how to avoid a trend of strong determinism in this case, since, to the extent that the future interferes with the present, our capability of changing the future becomes limited (Maudlin's arguments on this subject (see ref. [12]) still sound pertinent). As already mentioned (ref. [15]), I think that to discuss all alternative interpretations of quantum mechanics would lead us to deviate of our main point; furthermore, most probably I would not be able to present the arguments of their proponents in a correct and unbiased way. Please note that it is not my intention to put forward a new interpretation of the observed quantum mechanical phenomena and to compare it with other alternative interpretations. The purpose of this paper is to investigate quantum mechanics in a new experimental context. For divergent opinions on foundational questions on quantum mechanics see: Elegance and Enigma, ed M. Schlosshauer (Springer, 2011); see also M. Schlosshauer, J Kofler, and A. Zeilinger, arXiv:quant-ph/1301.1069, for the results of a poll carried out among participants of a conference on the foundations of quantum mechanics; and also C. Sommer, arXiv:quant-ph/1303.2719; and T. Norsen and S. Nelson, arXiv:quant-ph/1306.4646.

[22] A. Suarez and V. Scarani, Phys. Lett. A, 232, 9 (1997).

[23] M. F. Pusey, J. Barret, and T. Rudolph, Nature Physics, 8, 475 (2012).

[24] A. Bokulish, Reexamining The Quantum-Classical Relation (Cambridge University Press, 2008), for an interesting analysis of the interconnection between quantum and classical theories, including historic aspects.

[25] Naturally, even if, strictly speaking, $\mathbf{S}$ and $\mathbf{S}^{\prime}$ were not equivalent, Lorentz transformations would still be valid. For instance, when viewed from the preferred frame, light velocity would be constant, independently of the motion of the source, rods would contract along the direction of motion, and moving clocks would run slowly. Therefore, assuming that clocks were synchronized by means of standard procedures, using light signals, for instance, different moving frames would still be connected by a Lorentz transformation. Actually, there is some arbitrariness in the choice of the procedure we decide to use to synchronize clocks (this point of view is rooted in Poincaré's 
conventionalism: H. Poincaré, La Valeur de la Science (Flammarion, 1913); La Science et L'Hypothèse (Flammarion, 1906)). Superluminal signals (if they really exist) could be used instead, but would probably not be a good choice, since the covariance of the laws of physics would be lost. 